\title{
Seasonal Relapsing Minimal Change Disease: A Novel Strategy for Avoiding Long- Term Immunosuppression
}

\author{
Christopher Lawrence H. Terry Cook Liz Lightstone
}

Imperial Kidney and Transplant Centre, Imperial College Healthcare NHS Trust, Hammersmith Hospital, London, UK

Key Words

Minimal change disease $\cdot$ Tacrolimus $\cdot$ Corticosteroids

\begin{abstract}
Background: We describe the case of a young woman with seasonal allergic rhinitis who presented with signs of a lower respiratory tract infection, acute renal impairment and the nephrotic syndrome, demonstrated on biopsy to be due to minimal change disease (MCD) with acute tubular injury. Following initiation of high-dose corticosteroids, her respiratory symptoms and renal impairment improved, and the nephrotic syndrome went rapidly into remission, but relapsed, off treatment, in a seasonal fashion.
\end{abstract}

Management: In view of significant side effects related to corticosteroids, relapses were treated with the calcineurin inhibitor tacrolimus with excellent effect, but the patient was keen to avoid the complications of medium-term immunosuppression and so the drug was weaned early. She relapsed for the second time, whilst off tacrolimus, at the same time of year as at her initial presentation. In subsequent years we have successfully managed this patient with seasonal relapsing MCD with seasonal prophylactic tacrolimus therapy.

Discussion: We discuss the natural history of $M C D$ and treatment options and demonstrate the utility of a clear understanding of the natural history of the condition in order to predict disease relapse and tailor therapy to the individual patient.

\section{Case Report}

A 25-year-old Nigerian female pharmacist was admitted to our institution in June 2006 from a local hospital with the nephrotic syndrome. Two weeks prior to admission, she had been diagnosed with a lower respiratory tract infection and commenced on a 1-week course of amoxicillin. Two days after completing the antibiotic course, she noted oedema and oliguria. She presented to a local 
hospital which noted moderate renal impairment and proteinuria. She was transferred to our institution for assessment, diagnosis and treatment.

She had been in the United Kingdom for 10 years, had a past history of Malaria and took fexofenadine $120 \mathrm{mg}$ once daily for prophylaxis of seasonal allergic rhinitis. She was allergic to chloroquine.

Physical examination revealed fluid overload and haemodynamic stability (blood pressure 118/75 $\mathrm{mm} \mathrm{Hg}$ ). Auscultation of the praecordium revealed normal heart sounds. Coarse crackles were noted at the right lung base. Abdomen was soft and non-tender. Urinalysis revealed 4+ protein and 4+ blood.

Biochemical analysis revealed severe, and progressive, renal impairment, hypoalbuminaemia and hypercholesterolaemia. The urinary protein:creatinine ratio (PCR) was $2,377 \mathrm{mg} / \mathrm{mmol}$ (table 1). Immunological tests, including complement and lupus serology, were normal, or negative.

Chest radiograph was clear and a renal ultrasound scan showed normal sized, unobstructed kidneys (right $10.5 \mathrm{~cm}$, left $11.0 \mathrm{~cm}$ ). Doppler examination of the renal vessels excluded a renal vein thrombosis.

Renal biopsy (fig. 1) was obtained with an 18-gauge automatic spring loaded needle from the lower pole of the left kidney. Light and electron microscopy were performed. Light microscopy findings were of normal glomeruli and juxtaglomerular apparatus. The vessels were normal. Examination of the tubules revealed many with vacuolation of epithelial cell cytoplasm. Some tubules showed epithelial cell detachment and mitoses were present. Immunoperoxidase staining showed mesangial IgM+ and C1q+. Electron microscopy showed patent capillary loops lined by normal endothelium. There was a slight increase in mesangial matrix but no electron dense deposits. There was extensive effacement of the podocyte foot processes.

The histopathological diagnosis was consistent with minimal change disease (MCD) with associated acute tubular damage which was sufficient to explain the renal dysfunction at presentation.

The patient was commenced on corticosteroids (prednisolone $60 \mathrm{mg}$ once daily) along with bumetanide $2 \mathrm{mg}$ once daily; alfacalcidol $0.25 \mu \mathrm{g}$ once daily; enoxaparin $10 \mathrm{mg}$ subcutaneously once daily; lansoprazole $30 \mathrm{mg}$ once daily; atorvastatin $40 \mathrm{mg}$ every night; cetirizine $10 \mathrm{mg}$ once daily and nystatin $1 \mathrm{ml}$ four times a day. Renal function improved within days and she was discharged to outpatient follow-up.

Complete remission was achieved 4 weeks after presentation (albumin $>34 \mathrm{~g} / \mathrm{l}$ and urinary PCR $<50 \mathrm{mg} / \mathrm{mmol}$ ), but the patient experienced psychological disturbance on high-dose corticosteroids, including mania, insomnia, irrationality and inappropriate thought and speech. The dose of corticosteroid was halved with improvement in psychological symptoms. Over the next few weeks, she put on flesh weight and dual energy X-ray absorptiometry revealed osteopenia of the lumbar spine. A bisphosphonate was added to her therapy. After completing 6 months of high-dose steroids, the dose of prednisone was weaned to zero over 4 months finishing in April.

In June 2007, she developed respiratory symptoms, a productive cough and wheeze. She was not clinically nephrotic, but biochemical investigation confirmed relapse of the nephrotic syndrome with albumin $31 \mathrm{~g} / \mathrm{l}$, hypogammaglobulinaemia and urinary PCR of $779 \mathrm{mg} / \mathrm{mmol}$. After discussion with the patient, she was treated with the calcineurin inhibitor tacrolimus. Initially, the patient was concerned about the risk of diabetes but agreed that tacrolimus was a superior option to long-term corticosteroids because she had already developed undesirable side effects (i.e. osteopenia, striae and transient psychological disturbance). She was commenced on tacrolimus $3 \mathrm{mg}$ twice daily aiming for 12-hour trough levels of 4-6 ng/ml (by liquid chromatography mass spectrometry method) and within 4 weeks had again achieved a complete remission.

The patient's work as a pharmacist gave her an unusual insight into the treatment of her condition, and she was concerned about potential hazards of long-term tacrolimus, and so after 6 months of therapy, we agreed to wean the dose of tacrolimus by $2 \mathrm{mg}$ per month whilst warning the patient that we would normally treat for a longer duration and that she would be putting herself at risk of relapse. The patient discontinued tacrolimus in April 2008. 
In June 2008, the patient presented with the symptoms of a chest infection, treated for 1 week in the community with penicillin $V$ with no effect. On this occasion, she was clinically and biochemically nephrotic. In view of her previous rapid and sustained response to tacrolimus, this drug was recommenced at a dose of $4 \mathrm{mg}$ twice daily. Complete remission was again achieved after 4 weeks.

In view of the remarkably regular relapsing nature of this patient's nephrotic syndrome, presenting with respiratory symptoms, on a background of seasonal allergic rhinitis, we thought it most likely that disease relapses were related to atopy, rather than to the discontinuation of immunosuppression 2 months previously. Given her rapid response to treatment and the patient's reluctance to take long-term immunosuppression, we formulated a plan to give the patient tacrolimus during the summer months (between March and September). In March 2009, the patient electively commenced prophylactic tacrolimus $2 \mathrm{mg}$ twice daily, and this was up-titrated to achieve a trough level of $3.0 \mathrm{ng} / \mathrm{ml}$ by June 2009. In June 2009, the patient again experienced symptoms of a lower respiratory tract infection, but the urinary PCR never rose above $15 \mathrm{mg} / \mathrm{mmol}$ and albumin was maintained at $41 \mathrm{~g} / \mathrm{l}$. Tacrolimus was rapidly weaned in October 2009 and the seasonal approach to immunosuppression was repeated in 2010 and 2011. The patient has not experienced a relapse of MCD to date (fig. 2).

At the most recent follow-up (March 2012), the patient had normal renal function and albumin, and had undetectable levels of urinary protein excretion (table 1). We plan to continue this successful strategy for the foreseeable future.

\section{Discussion}

MCD is a common cause of the nephrotic syndrome in adults, after membranous glomerulonephropathy and focal segmental glomerulosclerosis [1]. The incidence of MCD is said to vary from 1 to 27 per million population. The aetiology of MCD is not fully understood, but the disease has been clearly associated with lymphomas, nonsteroidal anti-inflammatory drugs, and of particular relevance in this patient, appears to be more common in atopic individuals [2] or may be genuinely idiopathic.

The manifestations of the disease reflect a disorder of podocyte structure and function, the cause of which is uncertain. The Shalhoub hypothesis [3] suggests that there is a circulating permeability factor, caused by a disordered T cell compartment, and supporting evidence for this theory comes from the successful use of immunomodulatory therapy in the treatment of the disease [4].

No recent clinical trials exist of the treatment of MCD, although historical trials favour a daily dose of oral corticosteroids (over alternate day or intravenous corticosteroids) [5-7]. Other therapies are available: levimasole is commonly used in children; alkylating agents such as cyclophosphamide are effective but concerns exist about toxicity, potential for neoplastic change and infertility. The calcineurin inhibitors cyclosporin A [4] and tacrolimus [8] have also been used to good effect in frequently relapsing MCD, again most commonly in children. No clinical trial has yet been published investigating the use of tacrolimus to treat de novo MCD. The exact dose and duration of treatment (with either corticosteroids or calcineurin inhibitors) has not yet been established. MCD is often erroneously thought of as a benign disease; however, patients may present, as in this case, with acute kidney injury and it is a disease which commonly relapses such that patients may be repeatedly exposed to potentially toxic immunotherapy.

The pattern of relapse in this atopic patient, namely during the allergic rhinitis season, suggests that there is an immunological trigger to her nephrotic syndrome akin 
to a type 4 hypersensitivity reaction. It is tempting to suggest that the pollen to which the patient is allergic may share a common epitope with an antigen presented on the podocyte. Although less likely, it is possible that disease relapses were related to withdrawal of immunosuppression. Immunosuppression had been withdrawn approximately 2 months prior to each relapse.

Despite the probable immune-mediated trigger, it is not clear that tacrolimus is directly influencing the immune response, by inhibiting IL-2 transcription, as she still suffered from rhinitis but did not develop the nephrotic syndrome. There is, however, recent evidence that tacrolimus acts to protect and repair the podocyte (by stabilising the actin cytoskeleton [9]), thus directly preventing podocyte damage sufficient to prevent proteinuria.

An alternative strategy for this patient would have been to ask the patient to monitor for proteinuria with urine dipstick testing and commence tacrolimus in the event of developing proteinuria. Whilst this is certainly an option, the nephrotic syndrome can develop rapidly and may take 4 weeks or more to respond to immunotherapy. In addition, some patients with relapses of MCD may be difficult to treat and may require a combination of corticosteroids and tacrolimus, sometimes at high doses. Rather than react to the development of proteinuria (which is not a benign state resulting in acute kidney injury and a hypercoagulable state) we, with the patient's informed consent, prefer a proactive approach.

This case study reminds us of the lack of evidence-based medicine in the treatment of podocytopathies (such as MCD) but clearly demonstrates the usefulness of tacrolimus for MCD. By understanding the natural history of this patient's disease, we hope we have been able to demonstrate an unorthodox but effective management strategy which, importantly, is acceptable to the patient, thereby promoting concordance with therapy, and minimises exposure to potentially toxic agents. The wider role of tacrolimus in the treatment of MCD in adults has yet to be determined.

Table 1. Biochemical characteristics

\begin{tabular}{lllll}
\hline & $\begin{array}{l}2006 \\
\text { Presentation }\end{array}$ & $\begin{array}{l}\text { First } \\
\text { relapse }\end{array}$ & $\begin{array}{l}\text { Second } \\
\text { relapse }\end{array}$ & $\begin{array}{l}\text { March } \\
2012\end{array}$ \\
\hline Sodium, mmol/l & 132 & 137 & 137 & 138 \\
Potassium, mmol/l & 3.8 & 3.9 & 4.3 & 4.8 \\
Urea, mmol/l & 13.8 & 3.5 & 7.7 & 3.1 \\
Creatinine, $\mu \mathrm{mol} / \mathrm{l}$ & 233 & 82 & 99 & 60 \\
Albumin, g/l & 18 & 14 & 17 & 38 \\
Total Cholesterol, mmol/l & 7.4 & 6.3 & 7.9 & 4.3 \\
Urinary PCR, U & 2,377 & 1,017 & 1,439 & 0 \\
\hline
\end{tabular}




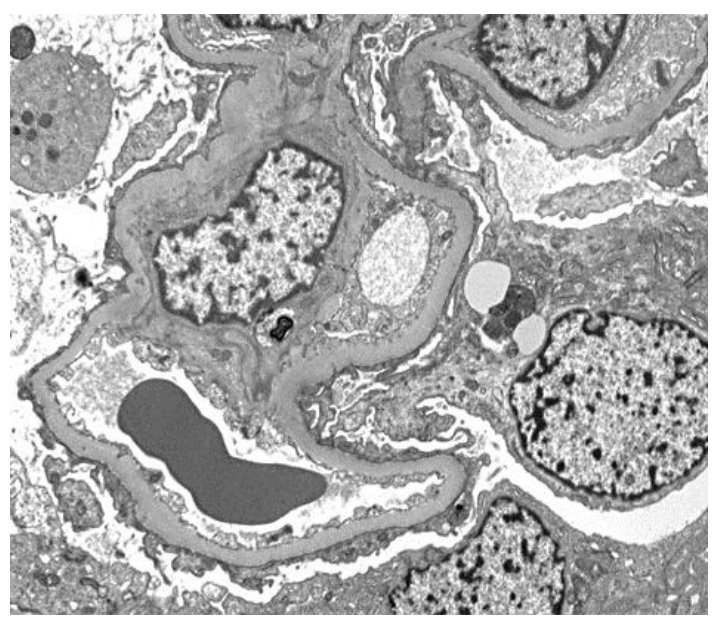

Fig. 1. Renal biopsy (electron microscopy) showing extensive fusion of the podocyte foot processes.
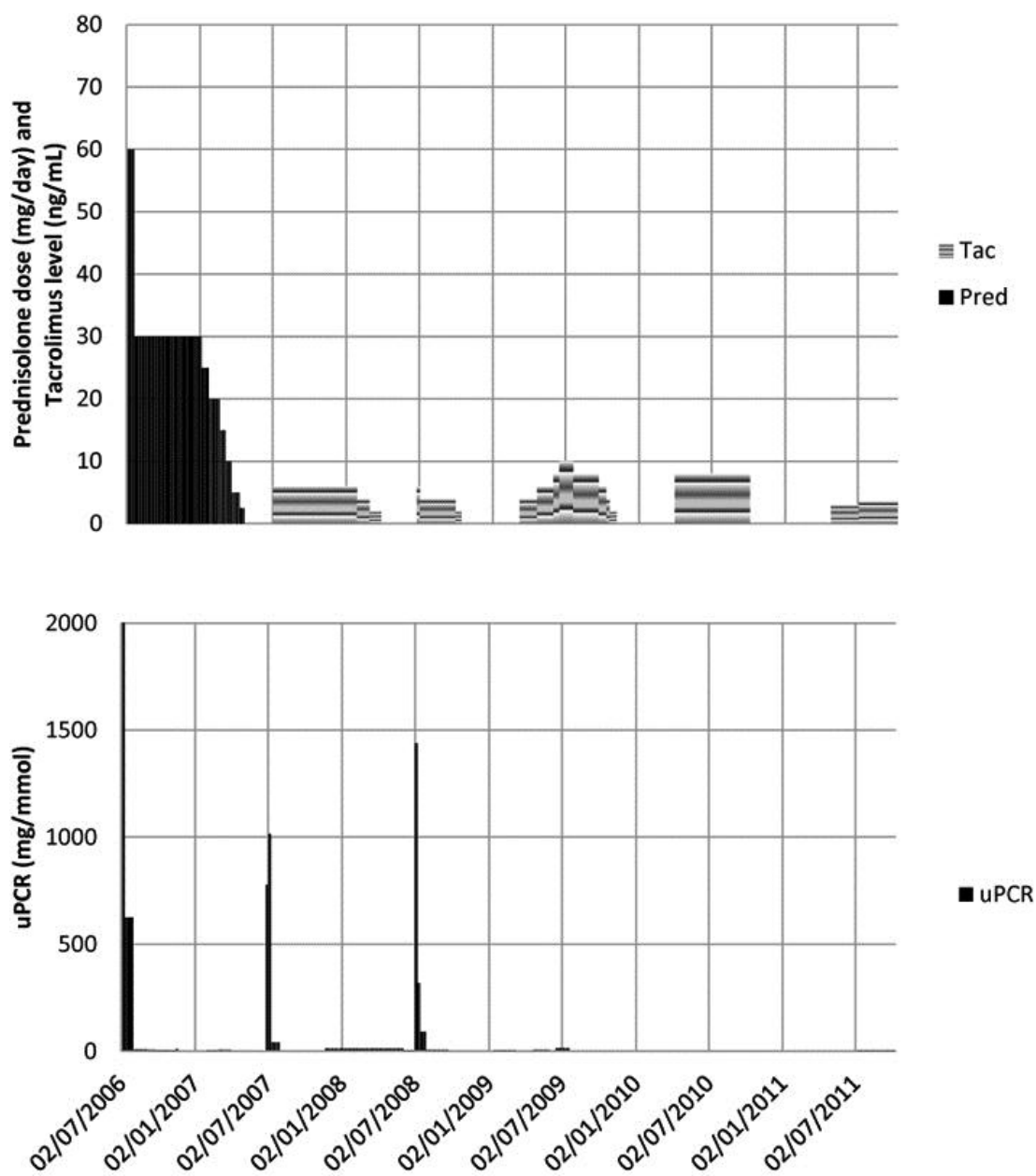

Fig. 2. Top panel showing prednisolone (Pred) dose (mg/day) in black and tacrolimus (Tac) level $(\mathrm{ng} / \mathrm{ml})$ in grey. Bottom panel showing urinary PCR (UPCR) $(\mathrm{mg} / \mathrm{mmol})$. Note that there has been no significant proteinuria since commencing prophylactic seasonal tacrolimus in 2009. 


\section{References}

1 Haas M, et al: Changing etiologies of unexplained adult nephrotic syndrome: a comparison of renal biopsy findings from 1976-1979 and 1995-1997. Am J Kidney Dis 1997;30:621-631.

$>2$ Meadow SR, Sarsfield JK: Steroid responsive nephrotic syndrome and allergy: clinical studies. Arch Dis Child 1981;56:509-516.

-3 Shalhoub RJ: Pathogenesis of lipoid nephrosis: a disorder of T-cell function. Lancet 1974;ii:556-560.

-4 Meyrier A: Treatment of idiopathic nephrosis by immunophillin modulation. Nephrol Dial Transplant 2003;18:vi79-vi86.

5 Black DA, Rose G, Brewer DB: Controlled trial of prednisone in adult patients with the nephrotic syndrome. BMJ 1970;3:421-426.

-6 Imbasciati E, Gusmano R, Edefonti A, et al: Controlled trial of methylprednisolone pulses and low dose oral predniosolone for the minimal change nephrotic syndrome. Br Med J (Clin Res Ed) 1985;291:13051308.

7 Yeung CK, Wong KL, Ng WL: Intravenous methylprednisolone pulse therapy in minimal change nephrotic syndrome. Aust N Z J Med 1983;13:349-351.

$>8$ Tang S, et al: Use of tacrolimus in steroid- and cyclophosphamide-resistant minimal change nephrotic syndrome. Am J Kid Dis 2003;42:E13-E15.

-9 Faul C, et al: The actin cytoskeleton of kidney podocytes is a direct target of the antiproteinuric effect of cyclosporine A. Nat Med 2008;14:931-938. 